\title{
Cluster Headache Patients Have Normal Circadian and Sleep Time Autonomic Nervous System Function
}

\author{
A Baharav ${ }^{1}$, Z Shinar $^{1}$, S Akselrod ${ }^{1}$, A Mosek $^{2}$, LR Davrath $^{1}$ \\ ${ }^{1}$ Tel-Aviv University, Tel-Aviv, Israel \\ ${ }^{2}$ Tel-Aviv Medical Center, Tel-Aviv, Israel
}

\begin{abstract}
Cluster headache $(\mathrm{CH})$ is a rare form of primary headache of neurovascular origin causing severe pain attacks associated with autonomic changes. Attacks are more likely to occur during sleep.

Time-frequency decomposition (TFD) of instantaneous Heart Rate Variability (HRV) is widely accepted as a non-invasive tool of investigation of autonomic nervous function and was applied in the present study.

Our goal was to estimate the autonomic features of $\mathrm{CH}$ patients and their connection to sleep.

The study included 20 subjects belonging to 3 groups: (a) $\mathrm{CH}$ (active headache attacks, N=7); (b) Normal control $(C, N=6)$; (c) patients with $\mathrm{CH}$ during a quiet period $(Q P, N=7)$

The study revealed similar circadian behaviour of all $H R V$ variables and of the HR in all groups indicating normal changes in central autonomic function between daytime and sleep in CH. Increased overall VLF power in $\mathrm{CH}$ compared to normal subjects suggests increased vasomotor activity during active headache periods only.
\end{abstract}

\section{Introduction}

Cluster headache is a relatively uncommon form of primary headache of neurovascular origin causing excruciating pain attacks [1]. Attacks are more likely to occur during sleep and sleep related breathing disorders are recognized as a possible trigger [2]. Autonomic changes such as lacrimation, conjuctival injection, nasal congestion and rhinorrhea are usually associated with attacks. Heart rate and blood pressure increase during spontaneous attacks.

Our goal was to estimate non invasively and with minimal intrusion into daily life, the Autonomic Nervous System (ANS) function of $\mathrm{CH}$ patients and its specific changes during sleep.

\section{Methods}

\subsection{Assessment of ANS function}

HRV analysis provides a widely recognized tool of non invasive investigation of ANS function. We chose to use the Time-frequency decomposition algorithm (TFD) previously developed in our laboratory [3]. This robust method computes the instantaneous power content as a 3 dimensional plane with varying time and frequency resolutions, chosen to provide optimal time resolution for each frequency. This method is suitable for non-steady state conditions. The frequency bands of interest are: (a) VLF between $0.005-0.04 \mathrm{~Hz}$ as a measure of vasomotor regulation and thermoregulation; (b) LF between 0.04$0.15 \mathrm{~Hz}$ representing both sympathetic and parasympathetic modulation in supine position, and mainly sympathetic one while standing; (c) HF between $0.15-0.5 \mathrm{~Hz}$ representing mainly parasympathetic modulation of the heart. The LF/HF ratio represents a generally accepted measure of the autonomic balance [4].

\subsection{Experimental procedure}

All subjects enrolled after informed consent and the study was approved by the local ethical committee. All subjects were examined by a neurologists who diagnosed the onset of a cluster headache period. These patients reported to the laboratory as soon as possible. All subjects were instrumented for $24 \mathrm{~h}$ Holter ECG recording and were instructed to complete a sleep diary while being connected to the recorder. The 12 lead ECG signal was digitized $(180 \mathrm{~Hz}$ sampling rate) for further analysis.

\subsection{Subjects}

Twenty nine male subjects enrolled in the study. Holter recordings from 9 individuals were excluded as they were exceedingly noisy or had arrhythmias for extensive periods of time, thus being unreliable. The remaining twenty subjects belonged to one of 3 groups: (a) $\mathrm{CH}: 7$ patients (age 43.3 \pm 7.1) during a period of headache attacks; (b) QP: 7 patients (age 41.4 \pm 7.9 ) with diagnosed $\mathrm{CH}$ during a quiet period; (c) C: 6 healthy subjects (age $38.6 \pm 7.5)$ with no chronic headache.

\subsection{ECG data analysis}

The first step of data analysis consisted in the automatic 
detection of $\mathrm{R}$ wave peaks, followed by visual inspection to ensure good quality signals and reject tracings that included sustained arrhythmias (such as frequent VPB, bigeminy etc), followed by manual correction of erroneous detections. Next, the RR intervals (RRI) were equally sampled to yield an RRI series for each 24 hour ECG tracing. Then time-frequency decomposition of the RRI series was performed by means of a previously published algorithm [3]. The results were integrated over the VLF, LF, and HF frequency ranges resulting in 3 series of integrals as a function of time. In addition $\mathrm{LF} / \mathrm{HF}$ and HR were also calculated as a function of time. Bedtime/sleep time (BT) was obtained from the sleep diary. Mean values for VLF, LF, HF, LF/HF, HR and were calculated for the entire $24 \mathrm{~h}$ recording period (WDWhole Day) and for BT. Figure 1 depicts a representative example of these parameters for one subject. Note the step change in RRI values during BT in Fig 1a. We further focused on the BT period, and specifically into periods with a low sympathovagal balance.

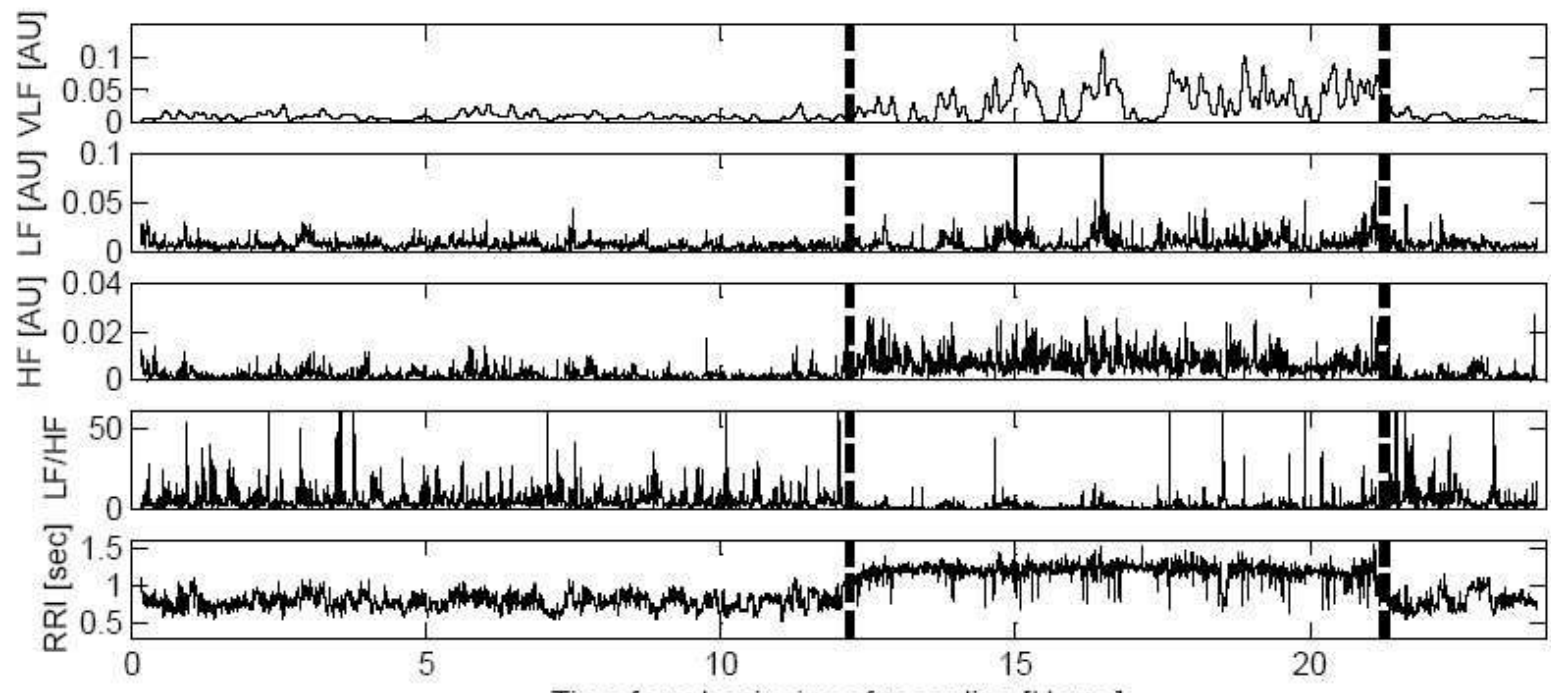

Time from beginning of recording [Hours]

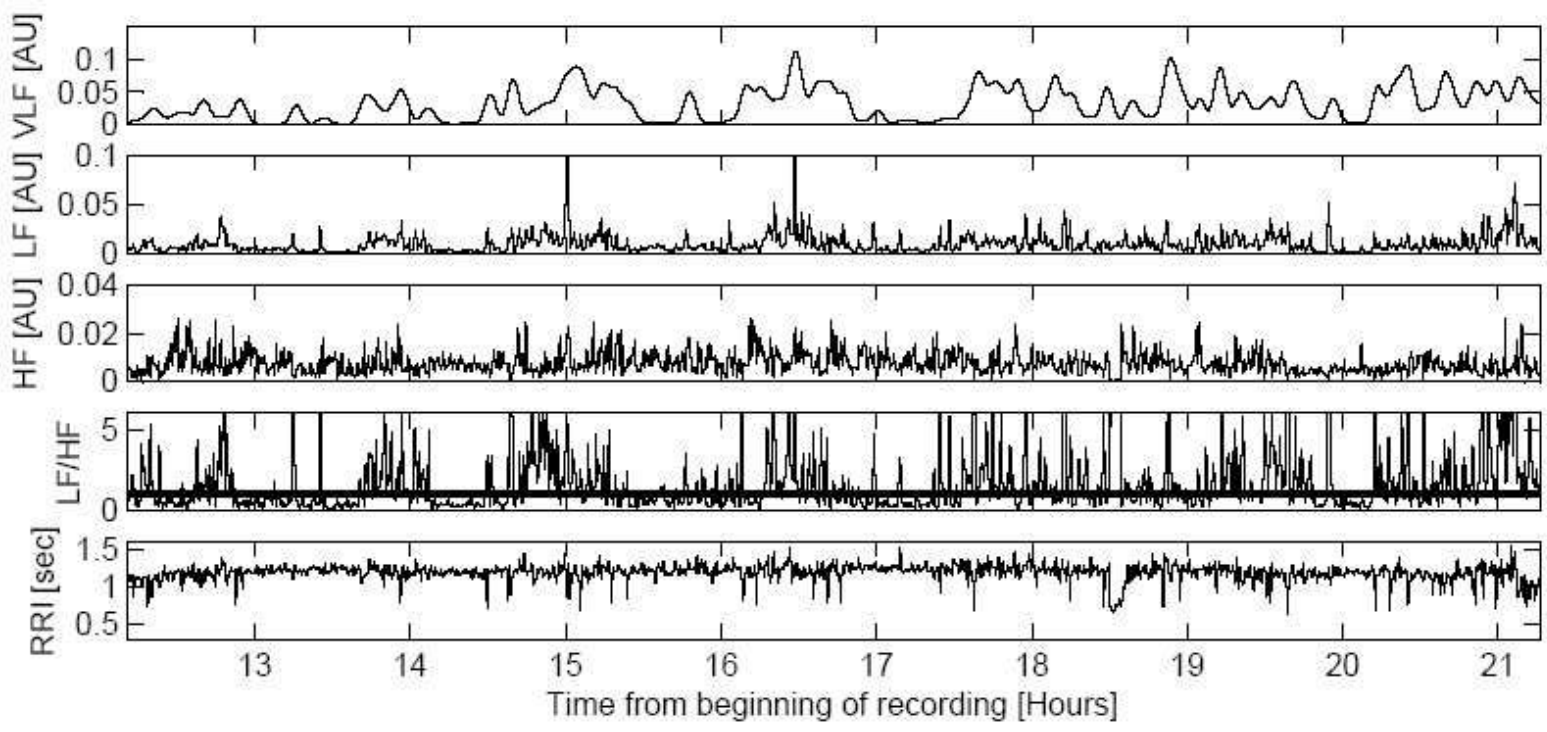

Figure 1: Autonomic variables and RR in one subject during the whole recording (a), and during the night (b). Vertical dashed lines in (a) delineate BT and horizontal line in (b) marks the threshold used to define presumed SWS. 
A threshold for this balance was individually obtained from the visual inspection of the parameters during BT and it was for each group: (a) QP: 1, 1, 1, 1, 2, 1, 2; (b) C: $1,1.5,1,0.5,0.25,2.5$; (c) $\mathrm{CH}: 1,1,1,1,1,1,1$. An example of the threshold level in one subject is presented in Fig 2b. According to previous studies [5] these periods were most probably epochs of Slow Wave Sleep (SWS). We calculated the mean values of all variables for the time intervals during BT when the autonomic balance did not reach the threshold, presumed SWS.

The last step in data analysis included visual inspection of the RRI as a function of time during BT aimed to detect periods of sleep disordered breathing [6].

\subsection{Statistical analysis}

Repeated measures ANOVA was applied to analyze the behavior of all spectral variables, autonomic balance and mean HR across WD, BT and SWS. One tailed paired t-test was used to compare the same variables within each of the studied groups, while unpaired t-test served to detect differences between the three groups. Differences were considered significant for $\mathrm{p}<0.05$ at a confidence level of $95 \%$.

\section{Results}

Subjects in the different groups were of similar age and their BMI did not differ significantly (QP: 28.2 2 2.7; $\mathrm{C}: 26.8 \pm 3.2 ; \mathrm{CH}: 26.4 \pm 2.2$ ). For clarity it is worthwhile to reemphasize that we examined 3 states: (a) WD- the entire recording time of about $24 \mathrm{~h}$; (b) BT-time in bed consisting mostly of presumed sleep and (c) SWSpresumed Slow Wave Sleep representing sub periods of BT with low autonomic balance.

The behavior of the studied variables over the 3 defined states during the study was similar as shown in Figures 2 and 3. However this behavior reached significance only for some of the variables:

(1) HR (see Fig 2a) decreased from WD to BT to SWS in $\mathrm{C}(\mathrm{p}=0.0001)$, and $\mathrm{QP}(\mathrm{p}=0.0006)$ but not for the $\mathrm{CH}$ group.

(2) LF/HF (see Fig 2b) was also lowest in SWS and highest during WD in all groups $(\mathrm{p}=0.0001)$.

(3) Spectral Variables (see Fig 3). VLF was higher during BT and SWS than during WD; it was lower during SWS than during BT. Although this behavior was similar in all groups it reached significance only for the QP group ( $p=0.033$ ). Both LF (Fig 3b) and HF (Fig 3c) changed during $\mathrm{BT}$ and SWS as compared to WD in all groups. LF showed a tendency to decrease from BT to SWS, whereas HF behaved the opposite, however these changes did not reach significance levels in any group.

(4) Between groups comparisons revealed that all variables studied were similar in QP and C groups. VLF,
LF and HF were significantly higher in $\mathrm{CH}$ patients than in normal controls during all states. No other significant differences were detected between groups, although a trend was observed for VLF, LF and HF to be lowest in controls, with similar levels in $\mathrm{QP}$ and $\mathrm{CH}$.

Based on manual estimation of sleep related breathing disorder by visual inspection of the RRI series 6 out of the 7 subjects in the QP group, 1 of 6 in the $\mathrm{C}$ group and 3 of 7 in the $\mathrm{CH}$ group were suspected to suffer from some degree of Obstructive Sleep Apnea (OSAS) [6]. Thus more than $60 \%$ of patients (either QP or $\mathrm{CH}$ ) and only $10 \%$ controls might suffer from OSAS.

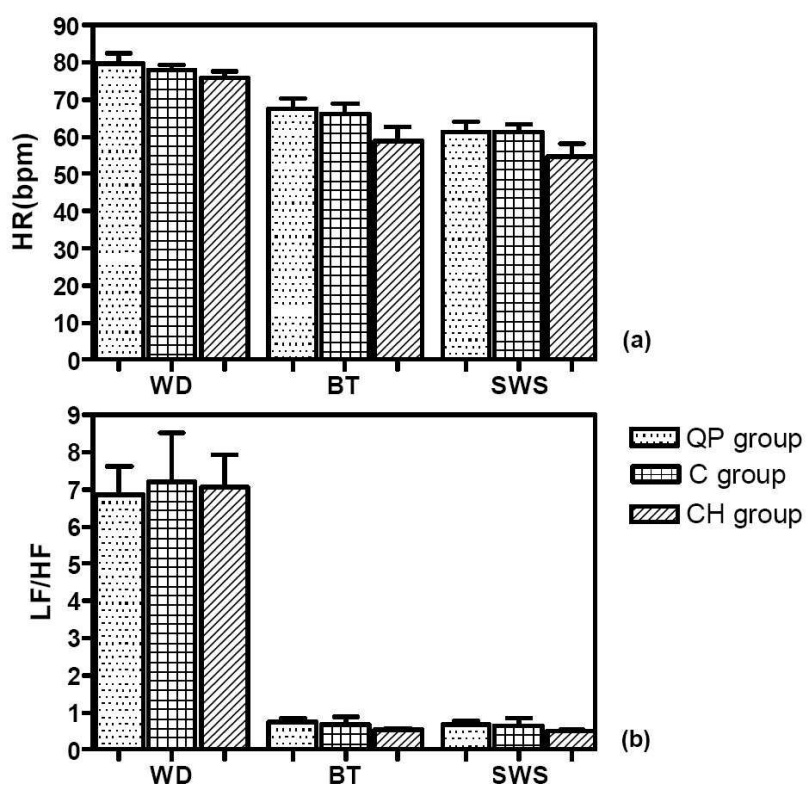

Figure 2: (a) Heart rate, and (b) autonomic balance during whole day (WD), bed time (BT), and presumed SWS in all groups.

\section{Discussion and conclusions}

The circadian behavior of LF, HF and autonomic balance was similar in all groups. This finding suggests that there is no basic underlying central autonomic dysfunction in patients suffering from recurrent attacks of $\mathrm{CH}$ during their quiet periods or during headache attacks. However we found that VLF, LF and $\mathrm{HF}$ were significantly higher in patients $(\mathrm{CH})$ than in normal controls when the entire recorded day was considered. No difference between these two groups was found during BT, SWS. No difference in the autonomic balance levels and behaviour was found between all subjects in all investigated states. Thus we may speculate that vasomotor changes accompany $\mathrm{CH}$ attacks as suggested by a higher VLF in $\mathrm{CH}$ as compared to normal control subjects, while the sympathetic balance was similar in both groups. 
Based on HRV analysis all patients, regardless of the presence/absence of active cluster headache, had a six times higher rate of suspicion for OSAS than normal controls. Occult sleep disordered breathing was previously described by Chervin et al [2] who also showed that in some patients, effective treatment of the OSAS with CPAP induced resolving of the $\mathrm{CH}$ attacks. High VLF was previously described in OSAS patients $[7,8]$, and resolved with treatment [9]. Thus, our findings suggest that there is a possible common autonomic function change underlying both $\mathrm{CH}$ and OSAS. Moreover the possible presence of OSAS in subjects with a history of $\mathrm{CH}$ was connected to their body weight, since BMI was slightly elevated but similar, in all patients and in normal controls.

$\mathrm{C}$ and $\mathrm{CH}$ represented two extremes in our study. Significant differences were detected between these two groups while QP did not differ significantly from either C or $\mathrm{CH}$.

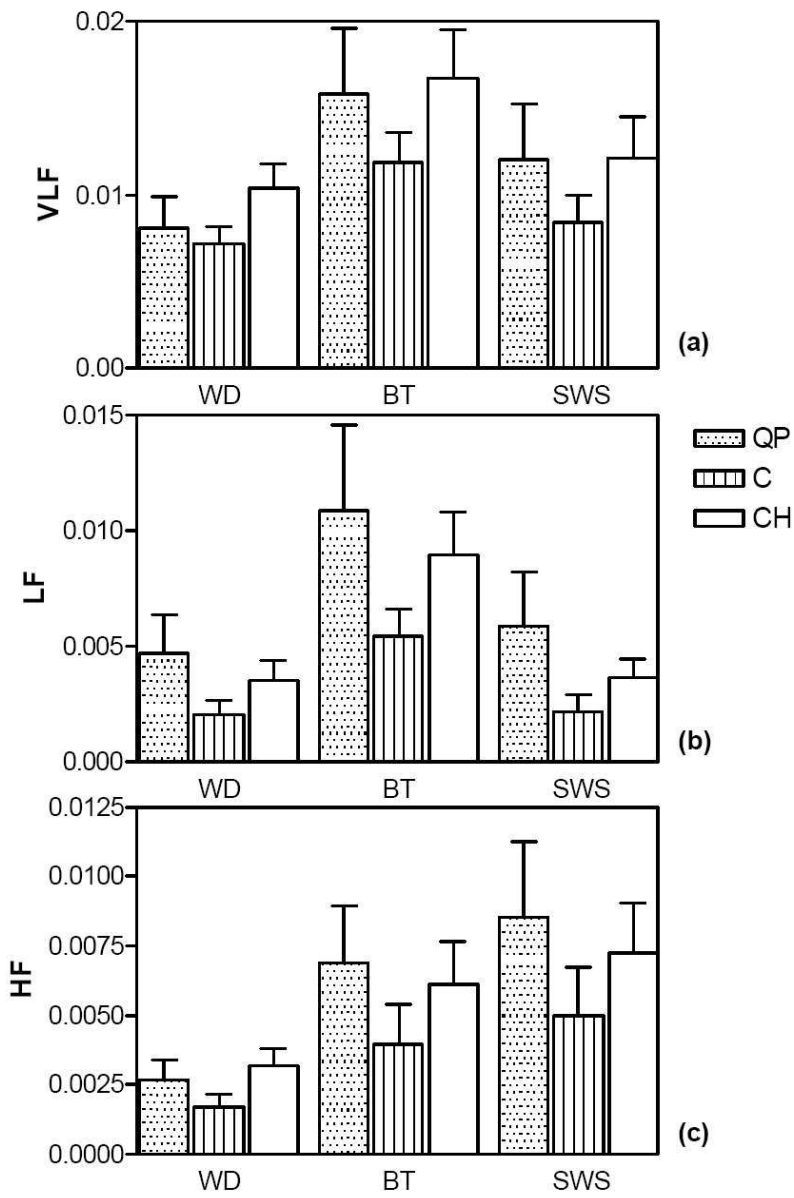

Figure 3: Spectral variables (a) VLF, (b) LF, and (c) HF, during whole day (WD), bed time (BT), and presumed SWS in all groups
Thus we may conclude that a significant increase in autonomic function, as detected from HRV analysis, occurs in patients only during the active headache period. During headache free time individuals with $\mathrm{CH}$ have not only normal circadian variation of autonomic function, but also similar levels of sympathetic, parasympathetic and vasomotor activity as normal individuals.

Although $\mathrm{CH}$ patients have normal circadian autonomic cardiovascular control, they have increased overall VLF frequency that might suggest vasomotor dysfunction. $\mathrm{CH}$ patients also appear to have an increased susceptibility to OSAS, and they display similar autonomic dysfunction detected by HRV analysis as patients with sleep related breathing disorder. Thus we might speculate that these disorders have some common underlying pathophysiological mechanisms. The results indicate a possible rationale for treatment during acute headache in patients, and they reconfirm the need to screen $\mathrm{CH}$ patients for occult OSAS.

\section{References}

[1] Goadsby PJ, Lipton RB. A review of paroxysmal hemicranias, SUNCT syndrome and other short-lasting headaches with autonomic feature, including new case. Brain 1997;120:193-209.

[2] Chervin RD, Zallek SN, Lin X et al. Sleep disordered breathing in patients with cluster headache. Neurology 2000;54:2302-2306.

[3] Toledo E., Gurevitz O., Hod H., Eldar M., Akselrod S. Wavelet analysis of instantaneous heart rate: a study of autonomic control during thrombolysis. Am J Physiol Regul Integr Comp Physiol., 284(4):R1079-91, 2003.

[4] Malliani A. Principles of Cardiovascular Neural Regulation in Health and Disease. Kluwer Academic, Boston, 2000.

[5] Shinar Z, Baharav A, Dagan Y, Akselrod S. Automatic detection of Slow Wave Sleep using Heart Rate Variability. Computers in Cardiology 2001;28:225-228.

[6] Shinar Z, Baharav A, Akselrod S. Obstructive sleep apnea detection based on the electrocardiogram. Computers in Cardiology 2000;27:267-270.

[7] Shiomi T, Guilleminault C, Sasanabe R, Hirota I, Maekawa M, Kobayashi T. Augmented very low frequency component of heart rate variability during obstructive sleep apnea. Sleep 1996;19:370-7.

[8] Narkiewicz K, Montano N, Cogliati C et al. Altered cardiovascular variability in obstructive sleep apnea. Circulation 1998;98:1071-1077.

[9] Khoo MCK, Belozeroff V, Berry RB and Sassoon CSH. Cardiac autonomic control in obstructive sleep apnea. Effects of long-term CPAP therapy. AM J Respir Crit Care Med 2001,164:807-812.

Address for correspondence:

Dr. Armanda Baharav

The Abramson Center for Medical Physics, Tel-Aviv University, Tel-Aviv, Israel.

E-mail address: armanda@netvision.net.il 